\title{
Estado del arte de las metodologías y modelos de los Objetos Virtuales de Aprendizaje (OVAS) en Colombia
}

\section{State of the art of Virtual Learning Object (VLO) models and methodologies in Colombia}

\section{Der neueste stand der methodologien und Beispiele Von Virtuellen Lernobjekten in Kolumbien}

\author{
Irlesa Indira Sánchez Medina \\ Magister en Educación. Universidad Cooperativa de Colombia. Neiva. Colombia. \\ irlesa.sanchez@campusucc.edu.co
}

\begin{abstract}
Resumen
Este artículo recopila las metodologías adoptadas y modelos implementados para la gestión de contenidos educativos digitales en el campo de los Objetos Virtuales de Aprendizaje (OVAs) por el Ministerio de Educación Nacional MEN, Universidades públicas y privadas de Colombia. Contribuye a fortalecer la capacidad de Innovación Educativa en el país, mostrando como se puede realizar una gestión efectiva y eficaz a través de estrategias basadas en la innovación, mediante el desarrollo de las etapas de formulación, diseño, desarrollo, uso, apropiación y producción de Objetos Virtuales de Aprendizaje como contenidos educativos digitales. Muestra sitios en la web donde la comunidad educativa puede encontrar los repositorios de Objetos Virtuales de Aprendizaje validados por el Ministerio de Educación Nacional MEN y las Universidades Colombianas.
\end{abstract}

Palabras claves: Objeto virtual de aprendizaje (OVA), metodología, enfoque, educación superior, Ministerio de Educación Nacional (MEN)

\begin{abstract}
This article compiles the adopted methodologies and approaches adopted for the management of digital educational content in the field of Virtual Learning Objects (OVAs) MEN by the Ministry of National Education, public and private universities in Colombia. Contribute to strengthening the capacity of Educational Innovation in the country, showing how you can make an effective and efficient management through based on innovation, by developing stages of formulation, design, development, use, ownership and production strategies Virtual Learning objects as digital educational content. Sample sites on the web where you can find educational community repositories of Learning Objects Virtual validated by the Ministry of National Education and MEN Colombian Universities.
\end{abstract}

Keywords: Virtual Learning Objects (OVAs), methodologies, higher education, Ministry of National Education(MEN).

\section{Zusammenfassung}

Dieser Artikel stellt angewandte Methodologien und bereits eingeführte Modelle von digitalen Lehrinhalten zusammen, die für für das Nationale Ministerium für Bildung (MEN), sowie öffentliche und private Universitäten in Kolumbien auf dem Feld der virtuellen Lernobjekte entwickelt wurden. Er trägt dazu bei, die Kapazität der edukativen Innovation im Land zu stärken, indem er zeigt, wie man eine effektives und effizientes Entwicklung durch Strategien, die auf Innovation beruhen, mit Hilfe von Planung, Design, Entwicklung, Anwendung, Aneignung und der Produktion von virtuellen Lernobjekten als digitale Lehrinhalte gestalten kann,. Er zeigt Webseiten auf, wo die Lerngemeinschaft Zusammenstellungen virtueller Lernobjekte finden kann, die vom MEN und den kolumbianischen Universitäten anerkannt worden sind. 


\section{Introducción}

Los avances tecnológicos han transformado a las naciones en sus ámbitos sociales, económicos, culturales y educativos.

En Colombia resulta evidente que las herramientas tecnológicas y el uso de las Tecnologías de la Información y Comunicación (TIC) en ambientes educativos juegan y jugarán un rol protagónico en el fortalecimiento de la capacidad de los sistemas educativos y en el mejoramiento de su calidad; razón por la cual es constante el impulso que desde el Ministerio de Educación Nacional se da para mejorar las condiciones y los servicios de la infraestructura tecnológica nacional y promover su apropiación y uso por parte de las comunidades educativas; inicialmente desde el Programa Nacional de Uso de Medios y TIC (2003 2011) y, actualmente, a través de la consolidación del Sistema Nacional de Innovación Educativa con Uso de TIC, que lidera la Oficina de Innovación Educativa con Uso de Nuevas Tecnologías. (Nacional. M. d., 2012)

Este artículo informativo presenta a toda la comunidad educativa colombiana las metodologías y/o modelos que se han desarrollado para la construcción de Objetos Virtuales de Aprendizaje (OVAs) desde las universidades públicas, privadas y el mismo Ministerio de Educación Nacional con el propósito de motivar a los lectores en su conocimiento y estudio, para que puedan contribuir a fortalecer la capacidad de Innovación Educativa en el país, que permitan ir cerrando la brecha de acceso a la información, al promover el acceso público y abierto al conocimiento; incentivar las capacidades nacionales para la producción, fortalecer los marcos de colaboración y cooperación para producción y gestión de contenidos educativos; consolidar la oferta nacional de contenidos educativos digitales y desarrollar la capacidad de acceso, uso y reutilización de los contenidos por parte de las comunidades educativas, evidenciado Plan Decenal de Educación (2006- 2016) y el Plan Sectorial de Educación 2010 - 2014 (Saavedra, 2012)

\section{Estado del arte}

En los últimos años han surgido varias iniciativas a nivel nacional e internacional, para disponer materiales digitales al servicio de la comunidad acadéimica, apoyando los procesos de enseñanza y aprendizaje. Algunos de estos trabajos están bajo la bandera de Repositorios o Bancos de Objetos de Aprendizaje (Learning Object Repositories). Los mecanismos de recolección y publicación de cada uno de estos trabajos son variados y dependientes del contexto en el cual se llevan a cabo. Un punto ausente en la mayoría de estas iniciativas es la colaboración interinstitucional para la producción, clasificación y publicación de objetos de aprendizaje. (Diego, 2012)

A continuación se presentan las metodologías y/o modelos con sus respectivas etapas, recomenda- ciones, pautas, relacionados con la construcción de Objetos Virtuales de Aprendizaje (OVAs) en Colombia, las cuales han dado pie a muchos de los Objetos Virtuales de Aprendizaje (OVAs), que se encuentran en los repositorios de las diferentes universidades del país y en el repositorio de la página (aprende, 2007) del Ministerio de Educación Nacional de la República de Colombia, en su portal educativo Colombia Aprende.

\subsection{Antecedentes}

El MEN llevó a cabo, durante el año 2005, el Primer Concurso Nacional de Objetos de Aprendizaje (OA), primer paso que realzó la necesidad de tener a disposición de la comunidad académica un número significativo de OA reunidos en un banco o repositorio ( (López Guzmán \& y García Peñalvo, 2004)), para ponerlos a disposición de toda la comunidad educativa nacional e internacional5 a través del Portal Educativo Nacional Colombia Aprende" (Mineducación, Plan nacional de desarrollo educativo., 2009) .

Paralelo a este concurso, se contemplaron los siguientes aspectos:

- El MEN adopta un modelo centralizado de recolección y publicación de objetos de aprendizaje, en un primer momento con los objetos del concurso.

- Todos los objetos publicados en el banco son gratuitos y de libre acceso. Esto llevó a los autores a ceder sus derechos patrimoniales.

Si bien el concurso tuvo muy buenos resultados, su seguimiento y posterior evaluación permitieron que afloraran algunos aspectos muy importantes que se debían tener en cuenta:

- La evaluación del material fue llevada a cabo por la Red Universitaria Mutis durante el concurso, sin embargo, esta fue una de las actividades que mayor demanda de tiempo y personal requirió, por lo tanto, al pensar en una estrategia que contemple un volumen de material significativamente mayor se debe reformular el proceso de evaluación. (Redmutis, 2014)

- Una de las condiciones del concurso era la cesión de derechos patrimoniales, esto influyó negativamente en el número de participantes, ya que era de esperarse que tanto instituciones como personas naturales no cedieran los derechos patrimoniales de los materiales, en los cuales habían invertido recursos humanos, físicos y económicos. Asimismo, hay un gran desconocimiento de las obligaciones contractuales del docente con la institución, por lo tanto, no se tenía claro quién poseía los derechos patrimoniales de los materiales.

Teniendo en cuenta estos aspectos, se vio claramente la necesidad de abordar, de una manera distinta, la estrategia, por esto, en el 2006 se generó un plan de acción que buscaba iniciar procesos de catalogación de OA en las Instituciones de Educación Superior (IES), 
estableciendo mecanismos de actualización y publicación que permitieran garantizar la sostenibilidad de cada uno de los bancos.

El MEN propició la ejecución del proyecto piloto "Catalogación de Objetos de Aprendizaje en IES", consolidando Bancos de Objetos de Aprendizaje dentro de algunas IES, líderes en el tema de la producción de material educativo digital. El proceso incluye la identificación de dicho material y su debida clasificación, mediante reglas y estándares comunes. (Colombia M. d., 2014)

Estos contenidos son recopilados, mediante el proceso de cosechado de los metadatos (Downes, 2003b) de los bancos institucionales, en un Banco Nacional de Metadatos de Objetos. Al mismo tiempo y como una estrategia complementaria se han venido desarrollando talleres presenciales de Uso de Objetos de Aprendizaje que buscan, por un lado, dar a conocer esta iniciativa, y por otro, avanzar en la estrategia general de uso, apropiación y producción de contenidos educativos. (Diego, 2012)

El concurso se realizó en diferentes categorías que abarcaban simuladores, cursos, tutoriales, y demás recursos que estimularan ambientes virtuales de aprendizaje (AVA). En esa ocasión el OA destacado fue el trabajo titulado: ¿Qué es un objeto de aprendizaje?, de la Universidad de Antioquia, este material presenta la información relacionada con OA desde su definición hasta su publicación, es un recurso valioso para quien desea conocer sobre este tema, trabajo que puede ser consultado en Universidad de Antioquia (Cuervo., Objetos de aprendizaje un estado del arte., 2011.).

Dado este primer paso para el 2007 el MEN decreta que todas las universidades del país deben tener un banco de objetos, en la cual indica las normas que deben cumplir dichos repositorios, tiene como objetivo "Aunar esfuerzos para que las Instituciones de Educación Superior (IES) inicien la catalogación y adaptación del material educativo digital que poseen, así como propiciar colaboración interinstitucional" (Cuervo, 2011).

A partir de lo anterior, las universidades emprenden un nuevo camino en el desarrollo de sus OA, encontrando diferentes obstáculos relacionado con la interpretación de objeto de aprendizaje, debido a la ambigüedad de la definición, estas experiencias están expuestas en el libro Objetos de Aprendizaje: prácticas y perspectivas educativas, realizado por la Universidad Pontificia Javeriana de Cali, (Cali, 2009) con el apoyo de otras universidades de carácter nacional, que fue lanzado oficialmente en febrero de 2010, las experiencias allí expuestas están sustentadas en los desafíos y retos que han tenido que vivir las universidades colombianas en la creación de OA, y en el enfoque que este término ha tomado en el transcurso de los años 2005 al 2009, debido a que el concepto ha sufrido una transformación importante desde que fue expuesto por primera vez por el MEN en el 2005, tanto en su concepto como en la forma de construcción.

El libro citado en el párrafo anterior promulga artículos tales como: "Herramientas para la búsqueda, catalogación, uso y evaluación de Objetos de Aprendizaje (OA). Una mirada desde la esperanza, lo esperado, lo prometido y lo posible", (Cuervo, 2011) que hablan sobre las ambigüedades que surgieron a la hora de su desarrollo y puesta en marcha, otros artículos generados hablan de metodologías de desarrollo de OA y experiencias educativas con esta nueva técnica, cada uno de los artículos realiza aportes importantes para conformar el concepto actual de objeto de aprendizaje, debido a que con la sinergia de sus experiencias en la parte pedagógica, metodológica y técnica, el MEN ha encontrado sus debilidades y fortalezas en este proyecto.

En un breve recorrido de las investigaciones realizadas sobre el tema se puede encontrar, que gran parte del trabajo se ha centrado en producción de material (OA) y en crear una metodología adecuada para su desarrollo.

Un ejemplo es el caso de la Universidad del Valle que "construyó un modelo de diseño de objetos que tiene inmerso un modelo de ciclo de vida de software, un modelo pedagógico, una propuesta de diseño gráfico y de integración de medios, lo que posibilita a los objetos ser reutilizables, interoperables y escalables" (Caldas, Una metodología para el diseño de objetos de aprendizaje. La experiencia de la Dirección de Nuevas Tecnologías y Educación Virtual, DINTEV, de la Universidad del Valle., 2010). Este proyecto fue sustentado con base en la experiencia que tiene la Institución en el desarrollo de material educativo desde hace décadas; por tal motivo la integración de los diferentes campos interdisciplinares fue un éxito, sin embargo una de las conclusiones de la investigación fue que el recurso educativo era dependiente del grupo interdisciplinar a la hora de ser actualizado, por ende no brindaba libertad al docente autor para realizar las actualizaciones como cualquier herramienta de autor , como eXelearning2, que le brinda autonomía al autor.

Se han propuesto otras metodologías, como la planteada por la Universidad Distrital Francisco José de Caldas, que se ha desarrollado en cinco fases comprendidas en: fundamentación técnica, diseño del $\mathrm{OA}$, desarrollo del $\mathrm{OA}$, implementación y análisis, las cuales son justificadas debido a que esta metodología fue desarrollada con el objetivo de integrar las TIC en el marco de un proyecto pedagógico de aula en las escuelas de los departamentos colombianos del Huila y del Tolima, dichas instituciones poseían limitaciones técnicas tanto de hardware como de software, al igual que el recurso humano capacitado en el tema de las TIC en la educación (Suarez, 2010).

Por su parte, la Universidad Pontificia Bolivariana con su propuesta Metova (Metodología para el diseño de 
Objetos Virtuales de Aprendizaje), sugiere tres fases de desarrollo, la primera es planeación conjunta, compuesta por recolección de requerimientos y lluvia de ideas para la gestación del proyecto, que es apoyada por un grupo de expertos de diseño, la segunda parte son propuestas didácticas e informáticas donde se realiza un diseño preliminar gráfico y se evalúa su aprobación, por último un mapa de navegación basado en una escritura de un guion (Cuervo, 2011).

Teniendo en cuenta otros trabajos realizados Eucario Parra Castrillón y Alexánder Narváez en el 2010 (Castrillón, 2010), (A., 2014), (Castrillón, "Revista Virtual Universidad Católica del Norte". No. 34, 2011) sobre el tema se encuentran proyectos en los cuales se desarrolló material para una determinada materia o carrera, ya sea de posgrado o pregrado, pero para uso privativo, ya que fue realizado para determinada comunidad académica, sin embargo la experiencia en la creación de Objetos de Aprendizaje (OA) es invaluable para el contexto educativo.

Actualmente el MEN, basado en las experiencias transcurridas desde el inicio del proyecto de integrar bancos de objetos en instituciones de educación superior, ofrece capacitaciones que están orientadas a corregir las debilidades del proyecto, aplicando los nuevos conceptos generados en las investigaciones realizadas por las principales universidades del país.

\subsubsection{Banco de Objetos virtuales de Aprendizaje - Colombia}

En el año 2006 a 2007 se creó y consolido entre el ministerio de educación nacional y las universidades colombianas el banco de objetos virtuales de aprendizaje. (salud., 2012)

El acceso al banco o repositorio de OVAs de cada una de las universidades se encuentra en la página web http://colombia.campusvirtualsp.org/?q=node/114.P or medio de esta plataforma usted podrá acceder a un sistema centralizado de catalogación estandarizado para recopilar información sobre materiales educativos digitales avalados por el G10 (grupo de 10 universidades colombianas - Universidad de los Andes, Universidad de Antioquia, Universidad Pontificia Bolivariana, Universidad EAFIT, Universidad externado de Colombia, Universidad Industrial de Santander, Pontificia Universidad Javeriana, Universidad Nacional de Colombia, Universidad del Norte, Universidad del Valle) en entornos virtuales. Dichos materiales pueden ser usados para apoyar los procesos de enseñanza-aprendizaje presencial y virtual, los cuales serán accedidos de forma pública y gratuita a nivel nacional e internacional por la comunidad académica.

A continuación se listan las universidades participantes y frente a cada una la dirección web donde se encuentra el repositorio de los objetos virtuales de aprendizaje que allí se han desarrollado con su respectivo metadato y estadísticas (visitas).
- Universidad de Antioquia -

http://aprendeenlinea.udea.edu.co/ova/?q=node\&page $=4$

- Universidad del Norte -

http://ylang-ylang.uninorte.edu.co:8080/drupal/.

- Universidad Pontificia Bolivariana -

http://eav.upb.edu.co/banco/.

- Universidad EAFIT -

http://www.ruana.edu.co/Paginas/banco-objetos-aprendizaje.aspx.

- Universidad Javeriana de Cali -

http://javevirtual.javerianacali.edu.co/component/phocagallery/cat egory/43-banco-de-objetos.

- Universidad del Valle -

http://objetos.univalle.edu.co/.

- Universidad Pedagógica y Tecnológica de Colombia

http://virtual.uptc.edu.co/drupal/.

- Universidad de la Sabana -

http://virtual.unisabana.edu.co/.

- Universidad de los Andes -

http://objetosvirtuales.uniandes.edu.co/.

- Universidad Nacional -

http://www.virtual.unal.edu.co/cursos/eLearning/oa_un/.

- Universidad Nacional Abierta y a Distancia.

http://repository.unad.edu.co/

- Universidad Minuto de Dios. -

http://repository.uniminuto.edu:8080/jspui/

En el año 2009 se incluyen nuevas universidades y corporaciones a los bancos de objetos de aprendizaje creados en el año 2006 y 2007, a saber:

- Universidad de Córdoba -

http://www.aves.edu.co/ovaunicor/.

- Universidad Industrial de Santander. -

http://www.uis.edu.co/webUIS/es/objetosAprendizaje.html

- Universidad Santo tomas -

http://boa.ustabuca.edu.co/.

- Universidad de Pamplona. -

http://www.unipamplona.edu.co/

- Universidad popular del Cesar. -

https://www.unicesar.edu.co/

- Universidad Politécnica de Cartagena.

http://repositorio.bib.upct.es/dspace/handle/10317/1287

- Eduteka. -

http://www.eduteka.org/OER.php

\subsubsection{Banco de Objetos en Latinoamérica}

PROTIC: (Repositorio Latinoamericano de Objetos de Aprendizaje) es una base de datos que pretende ser una respuesta a la escasez de información sobre proyectos TIC en la región y fomentar la participación de actores involucrados en este medio en su mantenimiento y mejoramiento permanente. Está orientada a los tomadores de decisión y a toda aquella persona que trabaje en el medio. http://www.protic.org/

LACLO: Comunidad Latinoamericana de Objetos de Aprendizaje. Es una comunidad abierta, integrada por personas e instituciones interesadas en la investigación, desarrollo y aplicación de las tecnologías relacionadas con Objetos de Aprendizaje en el sector educativo Latinoamericano. Su objetivo es articular los diferentes programas y proyectos que se han desarrollado en Latinoamérica para diseminar los avances y beneficios de esta tecnología, a fin de que en la región se puedan ofrecer recursos educativos personalizados y de calidad a cualquier persona, en 
cualquier momento y en cualquier lugar. http://www.laclo.org/

Biblioteca Itson (México): forma parte de un proyecto nacional para la producción de Objetos de Aprendizaje (OA), como parte del Fideicomiso SEP - UNAM (Secretaría de Educación Pública - Universidad Nacional Autónoma de México), con la finalidad de crear entre distintas universidades un repositorio de OA con carácter nacional -

http://biblioteca.itson.mx/oa/principal.htm

EUCARCHILE: Es un portal autónomo, pluralista y de servicio público que cuenta con la colaboración de los sectores público, privado y filantrópico. educarchile.cl contiene artículos, recursos clasificados por asignaturas, presentaciones, juegos educativos, imágenes y mucho más material que puede ser aprovechado para mejorar nuestra productividad dentro de las clases. http://www.educarchile.cl/ech/pro/app/search

\subsubsection{Banco de Objetos a nivel mundial.}

La iniciativa de Objetos de Aprendizaje a nivel mundial permite que cualquier docente o estudiante pueda acceder al material publicado en los diferentes bancos de Objetos de Aprendizaje (LOR - Learning Object Repository). Los siguientes enlaces conducen a algunos de los bancos más representativos y con mayor cantidad de objetos almacenados para el apoyo de las labores de enseñanza y aprendizaje.

JORUM: Jorum es un servicio de almacenamiento de recursos digitales en línea de libre acceso, que apoyen las labores de enseñanza - aprendizaje en las instituciones de educación Superior del Reino Unido, ayudando a construir comunidades que compartan y reutilicen materiales para la enseñanza y el aprendizaje. Ver sitio: http://www.jorum.ac.uk/

CAREO: (Campus Alberta Repository of Educational Objects) Su principal objetivo es la creación de una colección de material de aprendizaje multidisciplinario basado en web, que pueda ser fácilmente recuperado.

http://careo.ucalgary.ca/cgi-bin/WebObjects/CAREO. woa

MERLOT: Es un recurso abierto de libre acceso diseñado primordialmente para estudiantes y docentes de educación superior. Colecciona enlaces a materiales de aprendizaje en línea con anotaciones, revisiones y tareas. Ver sitio:

http://www.merlot.org/merlot/index.htm

The Maricopa Learning exchange (MLX): Es un repositorio digital de ideas, ejemplos y recursos (representados como paquetes) que apoyan el aprendizaje de los estudiantes vinculados a los colegios de la comunidad Maricopa.

http://www.mcli.dist.maricopa.edu/mlx/.
SMETE: Este portal educativo también ofrece servicios de librería digital dinámica para estudiantes y profesores. En este LOR se puede tener acceso a una gran riqueza de material para la enseñanza y aprendizaje, también se puede participar en esta comunidad de exploradores de todas las edades en ciencias, matemáticas, ingeniería y tecnología. Los estudiantes tienen acceso a recursos de ayuda para la preparación de clases o exámenes. Los profesores pueden encontrar materiales que se pueden usar en el aula de inmediato. SMETE abre los mundos de ciencia, matemática, ingeniería y la educación de tecnología a profesores y a estudiantes en cualquier momento y lugar en la sociedad de hoy. www.smete.org/smete/

Apple's Learning Exchange: Este sitio ha sido diseñado para promover el intercambio de ideas entre profesores, líderes educativos y personas innovadoras, brindando a los estudiantes modelos de mejores prácticas instruccionales. En Apple Learning Interchange los profesores encontrarán un listado de recursos dinámicos, diseñados para apoyarlos y mejorar la calidad de la enseñanza y el aprendizaje. http://www.apple.com/education/

LOLA Exchange: (Learning Objects-Learning Activities): Busca el intercambio para facilitar el acceso a objetos de aprendizaje de alta calidad. Contiene materiales que se pueden usar de manera transversal en el currículo con un énfasis particular en módulos que brindan a los estudiantes las habilidades necesarias para localizar, acceder y usar información significativa en la sociedad de hoy

http://www.colombiaaprende.edu.co/html/directivos /1598/article-99424.html

APROA: Aprendiendo con Repositorios de Objetos de Aprendizaje, es una iniciativa liderada por la Universidad de Chile, que busca crear una comunidad de docentes y estudiantes alrededor de la creación y utilización de objetos de aprendizaje en las actividades del aula, en particular cubriendo las áreas de agronomía y botánica. http://www.aproa.cl/

UNIVERSIA: La biblioteca Universia contiene 1.313 .990 recursos digitales entre los que se encuentran un número significativo de Objetos de Aprendizaje. http://www.universia.net/

CLOE: (Cooperative Learning Object Exchange) Es un repositorio en la Universidad de Waterloo, el cual revisa con atención el material publicado. Todos los objetos almacenados son revisados por parte de un grupo antes de ser publicados.

http://www.colombiaaprende.edu.co/html/directivos /1598/article-99424.html

OpenDOAR: Directorio de Repositorios de Acceso Abierto. http://www.opendoar.org/

ROAR: Registro de Repositorios de Acceso Abierto. http://roar.eprints.org/ 


\subsection{Marco Conceptual}

Definición de un Objeto Virtual de Aprendizaje.

Desde los años 60 se empezó a utilizar entre los programadores de computadores lo que se conoce como programación orientada a objetos, concepto que se trató de llevar al ámbito educativo mediante diferentes tipos de materiales diseñados bajo nuevas concepciones basadas en las ideas que fundamentan el paradigma de programación mencionado.

Estas ideas fueron tomadas para la formulación de lo que son los Objetos de Aprendizaje (OA). "El término Objetos de Aprendizaje fue popularizado por Wayne Hodgins en 1994 cuando nombró la CedMA (Computer Education Management Association) trabajando en el grupo Learning Architectures, APIs and Learning Objects'. CEdMA, describe sus propios propósitos como una provisión de un foro de discusión de cuestiones en entrenamiento por computador" (Friesen, 2004).

Se usa una metáfora para describir lo que se conoce como Repositorio de Objetos de Aprendizaje: "Un repositorio de objetos de aprendizaje es como un diccionario, un conjunto organizado de palabras que se pueden combinar fácilmente y volver a combinarse en múltiples y significativas frases" (Acker; Pearl et al., 2003), en cambio, los escépticos de esta nueva concepción de materiales educativos opinan que "los Objetos de Aprendizaje son simplemente un término de moda para hacer referencia a materiales, 'repositorio' como una biblioteca, 'metadatos' como un registro de un catálogo y 'secuencia estructurada' como un plan de estudios. Ellos concluyen que el conocimiento es una experiencia subjetiva en una comunidad de aprendices y no un objeto que puede transportarse hasta sus disciplinas" (ibid.).

Muchas definiciones de Objeto de Aprendizaje se han formulado, entre las que se cuentan la del profesor David A. Wiley II de la Utah State University:

Los Objetos de Aprendizaje son elementos de un nuevo tipo de instrucción basada en computadores enmarcada en el paradigma orientado a objetos de las ciencias de la computación. La orientación a objetos valora altamente la creación de componentes (llamados objetos) que pueden ser reutilizados en múltiples contextos. Esta es la idea fundamental detrás de los Objetos de Aprendizaje: diseños instruccionales que pueden construirse en pequeños componentes instruccionales (comparados con el tamaño de un curso completo), que pueden ser reutilizados un número de veces en diferentes contextos de aprendizaje. Adicionalmente, los Objetos de Aprendizaje son generalmente entendidos como entidades digitales para ser entregadas por Internet, significando que cualquier número de personas pueden acceder a ellos y usarlos simultáneamente (como oposición a los tradicionales medios de instrucción tal como un dispositivo de reproducción de video que solo puede existir en un lugar a la vez). Por otra parte, aquellos que incorporan objetos de aprendizaje pueden colaborar $y$ beneficiarse inmediatamente de las nuevas versiones. Estas diferencias significativas entre los objetos de aprendizaje y otros medios de instrucción han existido anteriormente. (Wiley D. , 2002)

Otra definición es la de Morales y García et al. (2005), quienes afirman que un OA es: "una unidad con un objetivo de aprendizaje, caracterizada por ser digital, independiente, con una o pocas ideas relacionadas y accesible a través de metadatos con la finalidad de ser reutilizadas en diferentes contextos y plataformas" (Erla Morales, 2004). Según Wiley (2003), "un Objeto de Aprendizaje es cualquier recurso digital que puede ser reutilizado para mediar en el aprendizaje (Wiley, 2003). Las dos principales metas detrás de la investigación y desarrollo de los Objetos de Aprendizaje son:

1. Mejorar el costo de la instrucción online, y

2. Habilitar la innovación pedagógica (Wiley, 2003).

Algo similar son los llamados Objetos Instruccionales de Gibbon, Nelson y otros:

A como nosotros usamos el término en este capítulo, los Objetos Instruccionales se refieren a cualquier elemento de una arquitectura que puede ser señalado independientemente dentro de un ensamblaje momentáneo en orden a crear un evento instruccional. El Objeto Instruccional puede incluir ambientes de problemas, modelos interactivos, problemas o conjuntos de problemas instruccionales, módulos de funciones instruccionales, rutinas modulares para el incremento instruccional (entrenamiento, retroalimentación, etc.), elementos de mensajes instruccionales, rutinas modulares para la representación de información, o módulos lógicos relacionados con los propósitos instruccionales (administración, registro, selección, etc.) (Gibbons, 2002).

En el Primer Concurso Nacional de Objetos de Aprendizaje "Un objeto virtual de aprendizaje se define como todo material estructurado de una forma significativa, asociado a un propósito educativo (en este caso para la Educación Superior) y que corresponda a un recurso de carácter digital que pueda ser distribuido y consultado a través de la Internet. El objeto de aprendizaje debe contar además con una ficha de registro o metadato consistente en un listado de atributos que además de describir el uso posible del objeto, permiten la catalogación y el intercambio del mismo." Definición de Objeto de Aprendizaje utilizada en el Primer Concurso Nacional de Objetos de Aprendizaje (Colombia, 2005).

Un Objeto Virtual de Aprendizaje, comúnmente llamado OVA, también se conoce en algunos contextos como OA, que significa Objeto de Aprendizaje, tomado de OL (en Inglés) Object Learning. (Uptc, 2014)

Aunque existen diferentes definiciones de un Objeto 
de Aprendizaje, en Colombia, expertos de diferentes universidades, por solicitud del Ministerio de Educación, construyeron un concepto propio para nuestro país, el cual está disponible en el portal Colombia Aprende:

Un objeto de aprendizaje es: "Un conjunto de recursos digitales, que pueden ser utilizados en diversos contextos, con un propósito educativo y constituido por al menos tres componentes internos: contenidos, actividades de aprendizaje y elementos de contextualización. Además, el Objeto de Aprendizaje, debe tener una estructura de información externa (metadato), para facilitar su almacenamiento, identificación y recuperación". (Uptc, 2014)

A su vez, se define un Objeto Informativo como: "Un conjunto de recursos digitales, que pueden ser utilizados en diversos contextos educativos, y que posee una estructura de información externa (metadato), para facilitar su almacenamiento, identificación y recuperación".

Y, un recurso digital: "Un recurso digital es cualquier tipo de información, que se encuentra almacenada en formato digital".

Así como la definición construida por el Equipo del Ministerio de Educación Nacional, en Colombia existen otras definiciones, como la del Instituto de Ingenieros Eléctricos y Electrónicos, IEEE:

"Un objeto es cualquier entidad digital o no digital, que puede ser usada, re-usada o referenciada, para el aprendizaje soportado en tecnología".

Aunque existe cierta similitud entre ambas definiciones, la diferencia sustancial radica, en que en el ámbito nacional, se ha enfocado a los Objetos Digitales o Virtuales. Además, se destacan los componentes que deben contener.

Según Morales Morgado (2007) quien propone unos criterios de evaluación para objetos de aprendizaje, clasificados como:

1. Criterios pedagógicos: categoría psicopedagógica, categoría didáctico-curricular.

2. Criterio de usabilidad: categoría diseño de interfaz y categoría diseño de navegación. (PORTILLA, 2014)

Del análisis de las anteriores definiciones sobre el significado de un OA, se puede concluir que los Objetos de Aprendizaje son recursos digitales disponibles en Internet que sirven para apoyar procesos académicos de gran valor pedagógico e instruccional distribuibles mediante redes con características claramente definidas que permiten ser referenciados para ser reutilizados usando tecnología y ensamblados en orden, para formar unidades de instrucción mayores con el único propósito de apoyar procesos educativos de enseñanza y aprendizaje.

\subsection{Metodologías y Modelos de Objetos Virtuales de Aprendizaje en Colombia.}

\subsubsection{Metodologías}

\subsubsection{Metodología de Diseño de Objetos de Aprendizaje de la Universidad del Valle}

La metodología para el diseño de objetos de aprendizaje de la Universidad del Valle está concebida en cinco fases, constituidas por una o varias etapas del modelo Iweb. Estas integran el modelo pedagógico, una propuesta de diseño gráfico y de producción y utilización de medios.

El Modelo Iweb se basa en aspectos como la planificación, el análisis, la ingeniería ó diseño e implementación, asociados a un marco tecnológico, pero aprovechando que la ingeniería de software demanda un proceso incremental y evolutivo. Este modelo se extiende en la etapa de ingeniería creando un ciclo asociado donde se establecen todas las bases de diseño del contenido, la interfaz gráfica, la navegación entre otras, convirtiendo el modelo clásico de espiral en un modelo eficaz para procesos de desarrollo en la web (mexico, 2012).

Las etapas de este modelo (según Presman, 2002 (Baddoo, 2009)) son:

a) Formulación: identificación de las metas y los objetivos.

b) Planificación: estima el costo global del proyecto y evalúa riesgos.

c) Análisis: establecimiento de los requisitos técnicos y de diseño, e identificación de los elementos del contenido que se van a incorporar.

d) Ingeniería: tiene dos tareas diseño del contenido y producción, en esta etapa se diseña, produce ó adquiere todo el contenido texto, gráfico y vídeo que se vaya a integrar al WebApp.

e) Generación de páginas: construcción haciendo uso de las herramientas para el desarrollo de WebApp, y se asocia con el diseño arquitectónico, de navegación y de interfaz para la elaboración de Web dinámicas.

f) Pruebas: se intentan descubrir errores y ayuda a asegurar que la WebApp funcionará correctamente en diferentes entornos (por ejemplo en diferentes navegadores).

g) Evaluación del cliente: Revisión de cada componente entregado en la WebApp. En este punto es donde se solicitan los cambios que se integraran en la siguiente ruta mediante el flujo incremental del proceso.

En resumen la metodología implementada para el desarrollo de $\mathrm{OA}$ en la Universidad del Valle se presenta a continuación (Meta, 2010). 
Tabla 1. Estructura de la metodología adoptada para el diseño de objetos de aprendizaje de la Universidad del Valle, Colombia.

\begin{tabular}{|c|c|c|c|}
\hline Fase & Etapa 1 & Etapa 2 & Etapa 3 \\
\hline $\begin{array}{l}\text { Formulación y } \\
\text { planificación }\end{array}$ & $\begin{array}{l}\text { Definición del proyecto: } \\
\text { * Conformación del grupo del trabajo. } \\
\text { * Delimitación del problema. } \\
\text { * Solución del problema. } \\
\text { * Los objetivos y productos del proyecto. } \\
\text { * Requerimientos funcionales y no } \\
\text { funcionales preliminares. } \\
\text { * Presupuesto. } \\
\text { * El cronograma. }\end{array}$ & & \\
\hline Análisis & $\begin{array}{l}\text { Diseño formativo: } \\
\text { * El público objeto. } \\
\text { * El enfoque pedagógico. } \\
\text { * Los objetivos. } \\
\text { * Estrategias de aprendizaje. } \\
\text { * El modelo de evaluación. } \\
\text { * } \text { Actividades de aprendizaje. } \\
\text { * Medios de comunicación, boceto. }\end{array}$ & $\begin{array}{l}\text { Primer análisis de } \\
\text { requerimientos } \\
\text { funcionales y } \\
\text { no funcionales. }\end{array}$ & \\
\hline Ingeniería & $\begin{array}{l}\text { Desarrollo de contenidos: } \\
\text { Uso de la hipermedia. } \\
\text { Hiperenlaces de contenido, glosarios, } \\
\text { contextualización e imagen. }\end{array}$ & $\begin{array}{l}\text { Análisis definitivo } \\
\text { de requerimientos } \\
\text { funcionales y no } \\
\text { funcionales. }\end{array}$ & $\begin{array}{l}\text { Diseño gráfico y } \\
\text { computacional. } \\
\text { * Diseños de } \\
\text { interfaz gráfica } \\
\text { páginas de inicio } \\
\text { y páginas } \\
\text { interiores. } \\
\text { * Selección de } \\
\text { texto, imágenes. }\end{array}$ \\
\hline $\begin{array}{l}\text { Generación } \\
\text { de páginas } \\
\text { y pruebas }\end{array}$ & Montaje de los objetos de aprendizaje. & $\begin{array}{l}\text { Publicacion y } \\
\text { pruebas internas. }\end{array}$ & Correcciones. \\
\hline $\begin{array}{l}\text { Evaluación } \\
\text { del cliente }\end{array}$ & Evaluación y corrección. & $\begin{array}{l}\text { Diseño y adición } \\
\text { de la versión lineal } \\
\text { del curso. }\end{array}$ & \\
\hline
\end{tabular}

Fuente: Borrero Caldas, María Clara. Cruz García, Edgar, Mayorga Muriel, Sandra y Ramírez González, Karen (2010) Una metodologla para el diseño de objetos de aprendizaje Dirección de Nuevas Tecnologías y jEducación Virtual,. DINTEV. De la Universidad del Valle Colombia. (Marla Clara Borrero, 2010)

\subsubsection{Metodología de Aprendizaje Colaborativo fundamentada en patrones para la producción y uso de Objetos de Aprendizaje MACOBA}

Analizando esta metodología, se puede manifestar que el eje central, está en torno al desarrollo de los recursos digitales, donde en primera instancia serán producidos (por maestros) y posteriormente consumidos (por aprendices) de forma colaborativa.

Las mejores prácticas de MACOBA para este proceso están resueltas con base en el paradigma de patrones: Al desarrollar un programa, normalmente, el desarrollador elige un paradigma de programación y algún patrón de diseño.
- Paradigmas de Programación: spaguetti, funcional, procedimental, modular, orientada a objetos, declarativo, orientado a la concurrencia, orientado a objetos.

- Patrones: creacionales, estructurales, comportamiento y sistema.

Así, los objetivos de la metodología MACOBA atienden a:

- Identificar buenas prácticas docentes en la aplicación de estrategias del aprendizaje colaborativo para facilitar la formación de personas mediadas por medio de elearning. 
- Determinar y documentar a manera de especificación los niveles del diseño de patrones para aprendizaje colaborativo.

- Crear un lenguaje de patrones para facilitar el diseño de aprendizaje colaborativo y desarrollar competencias colaborativas.

\subsubsection{Metodología MESOVA, Universidad Católica del Norte}

Es una metodología que se generó a partir de la problemática existente de crear objetos de aprendizaje a partir de prueba y error, sin pensar en un método que considere estructuradamente análisis de contexto, nivel educativo, ambientes apropiados o particularidades de las temáticas. Para los autores de la metodología, los objetos de aprendizaje son software y por lo tanto la propuesta se basa en metodologías existentes para producir software mediante el paradigma de la orientación a objetos. La metodología es completa, detallada y se basó en principios de calidad de software, lo que puede permitir la generación de productos confiables, pero que requieren de un equipo de producción de software para su desarrollo.

\subsubsection{Metodología de la Universidad Distrital Francisco José de Caldas}

Es un producto generado por el convenio entre la universidad y CPE (Computadores para educar), tomando como muestra instituciones educativas del Huila y Tolima, la propuesta se basó en el "diseño instruccional, software evolutivo con intenciones pedagógicas definidas, ajustados al contexto de escuelas" (comunicación., 2012).

Se resalta de esta metodología que es bastante rápida para implementar en la aplicación de creación de OA y que es sencilla para docentes que no han tenido mayor contacto con la tecnología.

Ilustración 1. Estructura de la metodología UBoa para la plataforma virtual de la Universidad de Boyacá

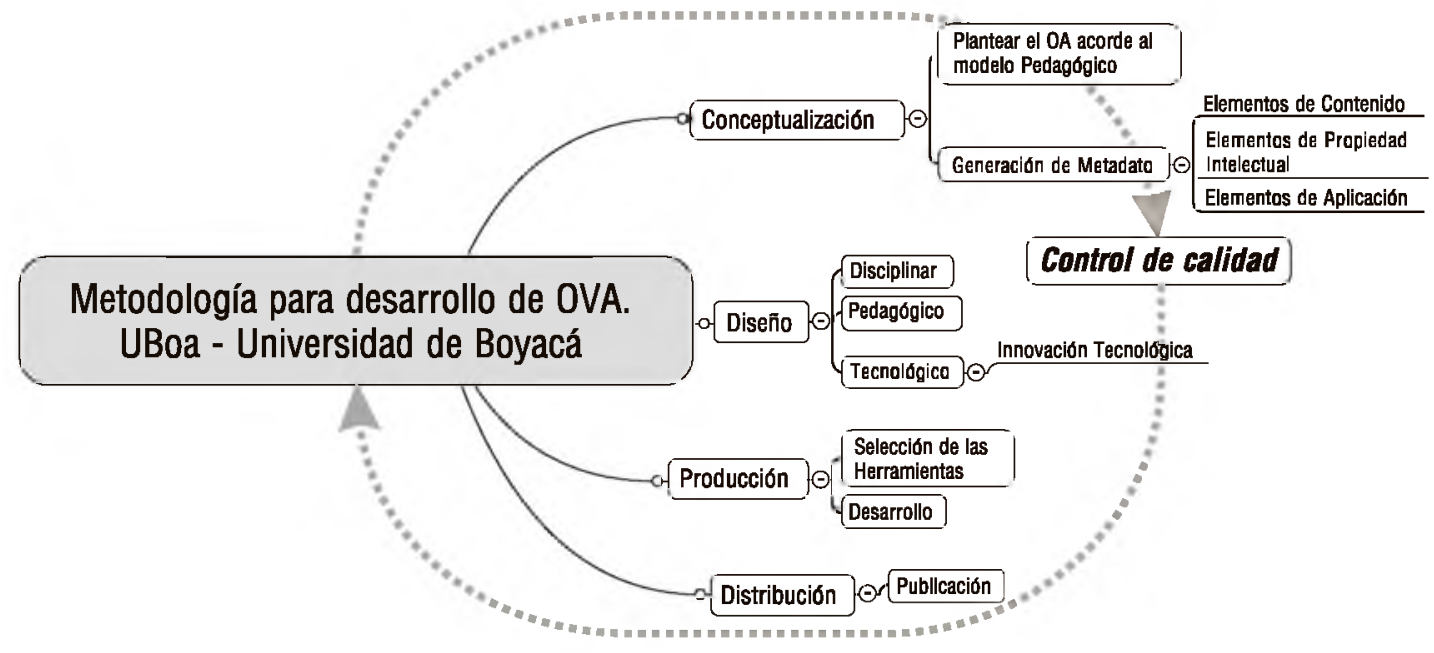

2.3.1.5 Metodología para el desarrollo de objetos de aprendizaje - UBoa de la Universidad de Boyacá

La integración de OA al proceso de enseñanza aprendizaje, permite ofrecer contenidos educativos que respondan a competencias específicas y permite al estudiante ser responsable de su aprendizaje, del mismo modo permite al docente estar capacitado en el uso de nuevas tecnologías. UBoa se convierte en un referente a la hora de planear y desarrollar objetos virtuales de aprendizaje aportando al proceso una organización interna, la identificación y selección de las competencias a desarrollar, así como el tipo de actividades cognitivas y su evaluación.

\section{Objetivos de la metodología UBoa}

La metodología UBoa se convierte en un proceso colaborativo que implementa estrategias de aprendizaje para la formación e-learning.

Los objetivos de la metodología UBoa son:

- Dar a conocer las diferentes fases del ciclo de vida para la construcción de OA así como los elementos básicos para la creación del metadato propio de los AO de la Universidad de Boyacá.

- Implementar la estructura u organización de contenidos de los OA para la plataforma virtual de la Universidad de Boyacá.

- Incorporar actividades de aprendizaje, evaluación activa e innovaciones tecnológicas en el desarrollo de los OA.

- Garantizar la calidad mediante la implementación de formatos de control de calidad en el diseño instruccional y diseño comunicacional de los $\mathrm{OA}$ en las diferentes fases de su desarrollo.

\section{Estructura de la metodología UBoa}

Tomando como referencia el ciclo de vida de un desarrollo de software, la metodología UBoa está estructurada con base en cinco fases, cada una con sus respectivas actividades y especificación de resultados. Estas son: Conceptualización, Diseño, Producción, Publicación y Control de Calidad. 


\subsubsection{Metodología para el diseño de Objetos Virtuales de Aprendizaje - Metova}

La metodología para la producción de Objetos Virtuales en la Universidad Pontificia Bolivariana con su propuesta, sugiere tres fases de desarrollo, la primera es planeación conjunta, compuesta por recolección de requerimientos y lluvia de ideas para la gestación del proyecto, que es apoyada por un grupo de expertos de diseño, la segunda parte son propuestas didácticas e informáticas donde se realiza un diseño preliminar gráfico y se evalúa su aprobación, por último un mapa de navegación basado en una escritura de un guion (Javeriana., 2009).

Del análisis de las anteriores metodologías para la elaboración de Objetos de aprendizaje se plantea la siguiente matriz DOFA.

\begin{tabular}{|c|c|}
\hline \multicolumn{2}{|c|}{ Matriz DOFA - Metodologías de Objetos Virtuales de Aprendizaje } \\
\hline Fortalezas & Debilidades \\
\hline $\begin{array}{l}\text { - Brinda a la comunidad educativa una guía de } \\
\text { como diseñar, desarrollar e implementar } \\
\text { Objetos Virtuales de Aprendizaje. } \\
\text { - Las metodologías son productos de proyectos } \\
\text { de investigación implementados en diferentes } \\
\text { campos del conocimiento científico. } \\
\text { - Gracias a las metodologías se han construido } \\
\text { bancos de repositorios de Objetos Virtuales de } \\
\text { Aprendizaje liderado por el Ministerio de } \\
\text { Educación Nacional en colaboración con } \\
\text { universidades privadas. } \\
\text { - Brinda a la comunidad educativa nuevas } \\
\text { alternativas de aprendizaje. } \\
\text { - Uso de herramientas disponibles en la web. }\end{array}$ & $\begin{array}{l}\text { - Es poca la información que se consigue acerca } \\
\text { de las metodologías desarrolladas en nuestro } \\
\text { país. } \\
\text { - Falta de sinergia entre las instituciones } \\
\text { dedicadas al desarrollos de Objetos Virtuales } \\
\text { de Aprendizaje para unificar metodología. } \\
\text { - Concientizar a la comunidad académica en } \\
\text { especial al cuerpo docente de la importancia } \\
\text { de utilizar Objetos de Aprendizaje enfocados a } \\
\text { los métodos de enseñanza B-Learning, E- } \\
\text { Learning y M-Learnig. } \\
\text { - Falta de infraestructura y tecnologica para } \\
\text { aplicar dichas metodologias. }\end{array}$ \\
\hline Oportunidades & Amenazas \\
\hline $\begin{array}{l}\text { - Crear una red en Colombia que se dedique solo } \\
\text { al diseño, desarrollo e implementaclón de } \\
\text { Objetos Virtuales de Aprendizaje. } \\
\text { - La existencia del Banco de Objetos de } \\
\text { Aprendizaje en página Colombia Aprende para } \\
\text { algunas áreas del conocimiento que los } \\
\text { profesores poco utilizan para apoyar su } \\
\text { proceso de enseñanza. } \\
\text { - Necesidad de una metodología funcional para la } \\
\text { creación de objetos virtuales de aprendizaje. }\end{array}$ & $\begin{array}{l}\text { - Diferentes tipos de metodologías atentan contra } \\
\text { la calidad de los Objetos de Aprendizaje. } \\
\text { - Comunidad académica inexperta en las } \\
\text { metodologías que gulan el trabajo con Objetos } \\
\text { Virtuales de Aprendizaje. } \\
\text { - La falta de recursos y tiempo para el diseño, } \\
\text { desarrollo e implementación de Objetos de } \\
\text { Aprendizaje innovadores. } \\
\text { - Variedad de metodologías de aprendizaje } \\
\text { virtual reconocidas. }\end{array}$ \\
\hline
\end{tabular}

\subsubsection{Modelos para \\ Objetos Virtuales de Aprendizaje.}

\subsubsection{Modelos objetos de aprendizaje (OA).}

Los objetos de aprendizajes, por sus características, plantean un desafío a docentes y diseñadores instruccionales, ya que su prototipo está orientado a la reutilización y deberían poder agruparse/ensamblarse para crear unidades de aprendizaje de mayor granularidad acorde al contexto educativo en el que se necesiten aplicarse. Como afirma Zapata Ros (2005) el diseño de los objetos de aprendizaje (OA) involucra tres disciplinas: diseño instruccional (brinda una s del marco para el diseño de materiales educativos), informática (el soporte tecnológico necesario para el desarrollo de este tipo de recursos) y bibliotecología (métodos y teoría de catalogación para el acceso, la clasificación, el almacenamiento y la búsqueda de recursos) (Astudillo, Informe de Tesis: Análisis del estado del arte de los objetos de aprendizaje., 2011).

Resulta evidente que enseñar y aprender desde la perspectiva de los objetos de aprendizaje exige nuevas formas de pensar y de hacer la enseñanza y el aprendizaje, dado que los diseños pedagógicos así como los desarrollos y procesos de enseñanzaaprendizaje han de plantearse de manera diferente". 
También plantea que para diseñar y utilizar OA, como para cualquier otro material educativo (Carneiro, 2012), es necesario tener en cuenta que:

$>$ Se debe pensar cuidadosamente los objetivos que se desean alcanzar.

$>$ Se deben seleccionar apropiadamente los contenidos.

$>$ Si los OA propician el aprendizaje y están bien diseñadas, el agruparlos y la forma en la que esto se hace, no necesariamente garantiza que el material obtenido continúe teniendo las mismas características.

$>$ Se deben analizar la granularidad del OA y evitar una excesiva fragmentación del saber.

> Para propiciar la reusabilidad de los OA deben ser fácilmente localizados aquí son centrales los metadatos.

$>$ Es fundamental que se integren los intereses pedagógicos, en virtud de que este aspecto no se debe dejar a cargo las herramientas utilizadas en la creación de los OA.

Los objetos de aprendizaje son elementos de un nuevo tipo de instrucción basada en computador y fundamentada en el paradigma computacional de 'orientación al objeto'. Se valora sobre todo la creación de componentes (objetos) que pueden ser reutilizados en múltiples contextos. Esta es la idea fundamental que se esconde tras los objetos de aprendizaje: los diseñadores instruccionales pueden construir pequeños componentes de instrucción (en relación con el tamaño de un curso entero) que pueden ser reutilizados varias veces en contextos de estudio diferentes. ( $\mathrm{La}$ casa, 2012.) Y la del Ministerio de Educación Nacional de Colombia que lo define como "un conjunto de recursos digitales que puede ser utilizado en diversos contextos, con un propósito educativo y constituido por al menos tres componentes internos: contenidos, actividades de aprendizaje y elementos de contextualización (Colombia., 2011).

\subsubsection{Modelo OA desde la teoría constructivista}

Para determinar el conjunto de requisitos que deben satisfacer los OVA es conveniente partir, por una parte, de las estrategias de aprendizaje que deben integrar, y por otra, de las potencialidades de su aplicación didáctica. Los entornos de aprendizaje constructivistas facilitan la elaboración del conocimiento a partir de la adaptación de los esquemas conceptuales a los modelos cognitivos de los estudiantes, propiciando un aprendizaje significativo (Moral, Diseñando Objetos de Aprendizaje como facilitadores, 2005).

Para atender a la diversidad cognitiva de los potenciales usuarios se precisa de entornos virtuales dinámicos y flexibles, en donde la metodología contemple variedad de actividades: estudios de casos, resolución de problemas en colaboración, toma de decisiones, prácticas de reflexión, análisis de múltiples interpretaciones, debates, trabajos colaborativos, etc. Los entornos virtuales deben facilitar al estudiante el acceso a contenidos educativos de calidad y permitirle seleccionar, clasificar, integrar e interrelacionar los más adecuados para la resolución de los problemas propuestos, y el logro de los objetivos. También deben contemplarse como paquetes de información multiformato, proporcionado de esta manera diferentes modos de presentación del contenido educativo, y por tanto, haciendo que la información llegue a través de distintas fuentes sensoriales, reforzando el aprendizaje.

Para que se constituyan en facilitadores del aprendizaje, los OVA deben concebirse como unidades mínimas de contenido didáctico con sentido por sí mismos, que sirvan de anclaje para aprendizajes posteriores. Así mismo, deben contener elementos altamente motivadores, interactivos, haciendo uso de la narrativa hipermedial que establezca relaciones que complementen la información a través de enlaces, y mapas conceptuales que presentan la información de una manera sintética y estructurada, priorizando la internavegabilidad.

\subsubsection{Modelo OA desde la teoría Instruccional.}

La teoría instrucción señala un modelo para desarrollar OVAs, esto permite a los desarrolladores definir las acciones a seguir en el proceso de generación de OVAs para ambientes virtuales de aprendizaje, atendiendo responsabilidades concernientes a la planeación y concertación, junto con los expertos en contenidos, de una serie de aspectos pedagógicos (objetivos de aprendizaje o competencias a desarrollar, actividades de aprendizaje, evaluación e integración con los contenidos) que garanticen un nivel de calidad adecuado del resultado final.

El desarrollador centra su atención en diseñar una actividad de aprendizaje central con características problemáticas, la cual proporciona sentido a los contenidos (objetos informativos). Dicha actividad de aprendizaje requiere de una previa formulación de objetivos de aprendizaje o competencias a lograr por el estudiante y podría estar acompañada por un esquema opcional de evaluación. De manera complementaria, construye elementos de "contextualización", que permiten la correcta identificación del objeto de aprendizaje como un todo integrado por quien lo revisa e interactúa con él. Elementos tan sencillos como un título o un logo institucional o más complejos como textos introductorios, de bienvenida, referencias bibliográficas o aspectos metodológicos son considerados elementos de contextualización. Un objeto Virtual debe diseñarse de acuerdo a unos juicios, Intemporalidad, no pierde vigencia en el tiempo, Usabilidad, es amigable para el usuario, Interacción, motiva al usuario a generar preguntas y a retornar respuestas. (Quiceno, 2009). 


\subsubsection{Modelos para la construcción de material educativo computarizado MEC}

El material educativo computarizado (MEC) es la denominación otorgada a las diferentes aplicaciones informáticas cuyo objetivo terminal es apoyar el aprendizaje. Se caracterizan porque es el alumno quien controla el ritmo de aprendizaje, la cantidad de ejercicios, decide cuando abandonar y reiniciar, interactuar reiteradas veces, en fin son muchos los beneficios. Por su parte el docente encuentra en ellos una ayuda significativa, pues en muchos casos en los MECs se registra toda la actividad del estudiante. De igual forma, el autor categoriza las diferentes aplicaciones informáticas MECs, de acuerdo con el objetivo que buscan, el momento educativo en que se vayan a utilizar o la complejidad en el diseño de los mismos (González, 2005).

A continuación en la tabla 2, se presenta el nombre de autor y denominación de la metodología acerca de los MEC y las etapas de cada una de ellas.

Tabla 2. Modelos metodológicos utilizados en la construcción de un MEC.

\begin{tabular}{|c|c|}
\hline Autor y denominación de la Metodología & Etapas \\
\hline $\begin{array}{l}\text { Álvaro Galvis Panqueva. } \\
\text { Metodología para el desarrollo de materiales } \\
\text { educativos computarizados. (Galvis, 1992) }\end{array}$ & $\begin{array}{l}\text { 1. Análisis de necesidades educativas } \\
\text { 2. Selección o planeación del desarrollo de MEC } \\
\text { 3. Ciclos para la selección o el desarrollo de MECs } \\
\text { 4. Diseño de MECs } \\
\text { 5. Entorno para el diseño del MEC } \\
\text { 6. Entorno del diseño } \\
\text { 7. Diseño educativo del MEC } \\
\text { 8. Diseño Computacional del MEC } \\
\text { 9. Preparación y revisión de los OA del MEC } \\
\text { 10. Desarrollo de MECs } \\
\text { 11. Desarrollo y documento del MEC } \\
\text { 12. Prueba piloto y Evaluación de MECs } \\
\text { 13. Prueba de campo de MECs }\end{array}$ \\
\hline $\begin{array}{l}\text { Ruffini. } \\
\text { Aproximación Sistemática y por etapas } \\
\text { utilizando sistemas multimedia de autor. } \\
\text { (Ruffini, 2000) }\end{array}$ & $\begin{array}{l}\text { 1. Análisis } \\
\text { 2. Seleccionar le Tópico a tratar } \\
\text { 3. Objetivos a cumplir } \\
\text { 4. Definir Proyecto } \\
\text { 5. Diseño de contenidos } \\
\text { 6. Diseño de hlpervínculos de navegación } \\
\text { 7. Evaluación del proyecto multimedia }\end{array}$ \\
\hline $\begin{array}{l}\text { Ovalle y Padilla. } \\
\text { para el desarrollo de un Software Educativo } \\
\text { Hipermedial (SEH) (J., 1998) }\end{array}$ & $\begin{array}{l}\text { 1. Análisis y estudio de factibilidad del proyecto. } \\
\text { 2. Diseño y esquematizaclón pedagógica de la aplicación. } \\
\text { 3. Desarrollo y programación. } \\
\text { 4. Distribución }\end{array}$ \\
\hline $\begin{array}{l}\text { Bertha López Azamar. } \\
\text { Gustavo Sergio Peláez Camarena. } \\
\text { Metodología para el desarrollo de Software } \\
\text { Educativo (DESED) (Azamar, 2006) }\end{array}$ & $\begin{array}{l}\text { 1. Determinar la necesidad de un S. E: } \\
\text { 2. Formación del equipo de trabajo } \\
\text { 3. Análisis y delimitación del tema } \\
\text { 4. Definición del usuario } \\
\text { 5. Estructuración del contenido } \\
\text { 6. Elección del tipo de software a desarrollar } \\
\text { 7. Diseño de interfaces } \\
\text { 8. Definición de las estructuras de evaluación } \\
\text { 9. Elección del ambiente de desarrollo } \\
\text { 10. Creación de una versión inicial } \\
\text { 11. Prueba de campo } \\
\text { 12. Mercadotecnia } \\
\text { 13. Entrega del producto final }\end{array}$ \\
\hline $\begin{array}{l}\text { Pere Marqués. } \\
\text { Metodología para la elaboración de } \\
\text { software educativo en Software Educativo. } \\
\text { (Marqués, 1995) }\end{array}$ & $\begin{array}{l}\text { 1. La génesis de la idea-semilla } \\
\text { 2. Pre-diseño o diseño funcional } \\
\text { 3. Estudio de viabilidad y marco del proyecto } \\
\text { 4. Dosier completo de diseño o diseño orgánico } \\
\text { 5. Programación y elaboración del prototipo alfa- test } \\
\text { 6. Redacción de la documentación del programa } \\
\text { 7. Evaluación interna } \\
\text { 8. Ajustes y elaboración del prototipo beta-test } \\
\text { 9. Evaluación externa } \\
\text { 10. Ajustes y elaboración de la versión } 1.0 \\
\text { 11. Publicación y mantenimiento del producto }\end{array}$ \\
\hline
\end{tabular}




\subsubsection{Modelo MEN Colombia Aprende.}

Según el portal educativo del ministerio de educación nacional, Colombia aprende, un objeto virtual de aprendizaje (OVA), se define como todo material estructurado de una forma significativa, asociado a un propósito educativo (en este caso para la Educación Superior) y que corresponda a un recurso de carácter digital que pueda ser distribuido y consultado a través de la Internet. El objeto de aprendizaje debe contar además con una ficha de registro o metadato consistente en un listado de atributos que además de describir el uso posible del objeto, permiten la catalogación y el intercambio del mismo." Definición de Objeto de Aprendizaje utilizada en el Primer Concurso Nacional de Objetos de Aprendizaje (Saavedra, 2012).

Teniendo en cuenta la evolución en la temática y la experiencia del país en el tema, se ha adoptado la siguiente definición. En tal sentido, dicho objeto debe diseñarse a partir de criterios como:

$>$ Atemporalidad: Para que no pierda vigencia en el tiempo y en los contextos utilizados.

> Didáctica: El objeto tácitamente responde a qué, para qué, con qué y quién aprende.

> Usabilidad: Que facilite el uso intuitivo del usuario interesado.

$>$ Interacción: Que motive al usuario a promulgar inquietudes y retornar respuestas o experiencias sustantivas de aprendizaje.

> Accesibilidad: Garantizada para el usuario interesado según los intereses que le asisten.

El estudio de las metodologías y enfoques para el diseño de Ovas fue útil para decidir las etapas que lleva el diseño, a continuación se presenta la formulación del prototipo.
Elementos estructurales de un OVA. El valor pedagógico de un OVA está presente en la disponibilidad de los siguientes componentes (ver ilustración 2).

Ilustración 2. Estructura general de un OVA.

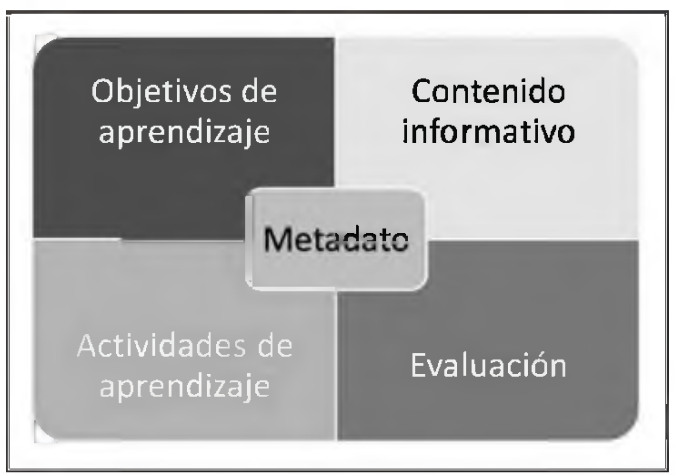

- Objetivos: Expresan de manera explícita lo que el estudiante va a aprender.

- Contenidos: Se refiere a los tipos de conocimiento y sus múltiples formas de representarlos, pueden ser: definiciones, explicaciones, artículos, videos, entrevistas, lecturas, opiniones, incluyendo enlaces a otros objetos, fuentes, referencias, etc.

- Actividades de aprendizaje: Que guían al estudiante para alcanzar los objetivos propuestos.

- Evaluación: es una herramienta que permite verificar el aprendizaje logrado. Están en concordancia con los objetivos propuestos y por el tipo de contenido presentado.

- Flementos de contextualización - Metadato: Que permiten reutilizar el objeto en otros escenarios, como por ejemplo los textos de introducción, el tipo de licenciamiento y los créditos del objeto.

Del análisis de los anteriores modelos para la elaboración de Objetos Virtuales de Aprendizaje podemos plantear siguiente matriz DOFA.

\begin{tabular}{|c|c|}
\hline \multicolumn{2}{|c|}{ Matriz DOFA de los modelos Objeios Virtuales de Aprendizaje } \\
\hline Fortalezas & Debilidades \\
\hline $\begin{array}{l}\text { - Brinda a la comunidad educativa modelos para el } \\
\text { desarrollo de objetos virtuales de aprendizaje. } \\
\text { - La teoria constructivista y humanística fortalecen los } \\
\text { productos implementados en diferentes campos del } \\
\text { conocimiento cientifico. } \\
\text { - Gracias a los modelos se ha logrado estandarizar la } \\
\text { producción de bancos de repositorios de Objetos } \\
\text { Virtuales de Aprendizaje. } \\
\text { - Los modelos permiten crear objetos de aprendizaje para } \\
\text { apoyar procesos pedagógicos. } \\
\text { - Avances tecnologicos-herramientas disponibles en la web. }\end{array}$ & $\begin{array}{l}\text { - Es escasa la información relacionada con los modelos } \\
\text { para el desarrollo de objetos de aprendizaje en nuestro } \\
\text { país. } \\
\text { - Compromiso por parte de las instituciones dedicadas } \\
\text { al desarrollos de Objetos Virtuales de Aprendizaje. } \\
\text { - Sensibilizar a los profesores sobre la importancia de } \\
\text { conocer el modelo aplicado en el desarrollo de los } \\
\text { Objetos de Aprendizaje. } \\
\text { - Capacitación a profesores en el manejo de } \\
\text { herramientas tecnológicas - uso de TIC. }\end{array}$ \\
\hline Oportunidades & Amenazas \\
\hline $\begin{array}{l}\text { - Capacitar a los profesores en el diseño, desarrollo e } \\
\text { implementación de Objetos Virtuales de Aprendizaje - } \\
\text { uso de TIC en educación. } \\
\text { - El profesor sera capaz de identificar la herramienta } \\
\text { tecnológica apropiada para apoyar sus clases de acuerdo } \\
\text { al modelo pedagógico institucional. }\end{array}$ & $\begin{array}{l}\text { Diferentes tipos de modelos afectan el uso y } \\
\text { aplicación de los Objetos de Aprendizaje. } \\
\text { - Desconocimiento de modelos pedagogicos que son la } \\
\text { base para el desarrollo de Objetos Virtuales de } \\
\text { Aprendizaje. } \\
\text { - Variedad de modelos de aprendizaje virtual } \\
\text { reconocidos. }\end{array}$ \\
\hline
\end{tabular}




\section{Conclusiones}

El aprendizaje de un estudiante no depende de la modalidad educativa en que se encuentre, sino de cómo se le presentan los contenidos temáticos, de las actividades que refuerzan su aprendizaje y de la coherencia que exista entre el material educativo con sus necesidades y objetivos educativos. Es en este punto en donde as metodologías y/o enfoques para la construcción de OVAs juegan un papel importante debido a su forma de presentar contenidos y de transferir conocimientos; esto quedó registrado en cada una de las metodologías $\mathrm{y} / 0$ enfoques consultadas y presentadas en este artículo.

Desde la pedagogía un OVA debe poseer los siguientes componentes: Titulo, palabras claves, objetivos de aprendizaje (competencias), contenido informativo, actividades de aprendizaje, ejemplos, retroalimentación, evaluación y un elemento fundamental en el uso de los objetos de aprendizaje el metadato, que permite realizar la búsqueda rápida y precisa del objeto, para identificarlo, almacenarlo y distribuirlo, como lo mencionó MEN.

Para lograr la creación de OVAs con calidad, se han desarrollado e implementado un conjunto de estándares y/o especificaciones que contribuyen en la realización de estos objetos, entre los que se destacan estándares para la creación de objetos de contenido formativo estructurado y estándares para la creación de metadatos. Estos estándares permiten establecer tanto la estructura interna de los objetos como la forma adecuada de almacenarlos y distribuirlos.

Un Ova es un material digital de aprendizaje diseñado a través de recursos tecnológicos centrado en las necesidades de los estudiantes cuyo propósito es educativo $y$ formativo, estructurado de forma significativa que sirve para adquirir conocimientos específicos, desarrollar competencias particulares y que se puede consultar por internet, atendiendo las características de los estudiantes y sus estilos de aprendizaje.

El profesor, hoy tiene que ser un profesional integral, cuyas competencias son de orden tecnológico, actualizado en sus saber especifico, capacitado en Pedagogía y didáctica de la virtualidad, Competencia técnica y tecnológica, y Diseño instruccional, para poder ser un actor en el diseño de OVAS.

\section{Referencias Bibliográficas}

A., J. M. (2014, 2 1). Matemáticas y TIC. Ambientes virtuales de aprendizaje en clase de Matemáticas. Retrieved 46 , 2014, from Matemáticas y TIC. Ambientes virtuales de aprendizaje en clase de Matemáticas.:

http://www.virtualeduca.org/ponencias2014/14/Matematicas yTIC.Ambientesvirtualesdeaprendizajeenlaclasedematematicas. pdf

Aprende, C. (2007, 4 21). Objetos virtuales de aprendizaje e informativos. Retrieved 4 6, 2014, from Objetos virtuales de aprendizaje e informativos: http://www.colombiaaprende.edu.co/html/directivos $/ 1598$ /propertyvalue-34418.html

Astudillo, G. J. (2011). Análisis del estado del arte de los objetos de aprendizaj. Argentina: Facultad de Informática - Universidad Nacional de La Plata.

Astudillo, G. J. (2011). Informe de Tesis: Análisis del estado del arte de los objetos de aprendizaje. Argentina.: Facultad de Informática Universidad Nacional de La Plata .

Azamar, G. P. (2006). Metodología para el Desarrollo de Software Educativo (DESED). UPICSA, 41-42.

Baddoo, R. V. (2009). Software Process Improvement. . Dublin: Editorial Springer.

Caldas, M. C. (2010). Una metodología para el diseño de objetos de aprendizaje. La experiencia de la Dirección de Nuevas Tecnologías yEducación. Revista Cientifica Universidad del Valle, 13.

Caldas, M. C. (2010). Una metodología para el diseño de objetos de aprendizaje. La experiencia de la Dirección de Nuevas Tecnologías y Educación Virtual, DINTEV, de la Universidad del Valle. Cali: Universidad del Valle.

Cali, P. U. (2009). Objetos de aprendizaje - prácticas y perspectivas educativas. Cali.: Multimedios - PUJ-Cali. ISBN: 958-8162-65-3.

Carneiro, R. (2012). Los desafios de las TIC para el cambio educativo. España.: Fundación santillana. ISBN: 978-84-7666-197-0.

Castrillón, E. P. (2010, 12 2). "Revista Virtual Universidad Católica del Norte". No. 31. Retrieved 4 5, 2014, from "Revista Virtual Universidad Católica del Norte". No. 31 : file://C:/Users/PRINCIPAL/Downloads/38-201-2-PB\%20(1).pdf

Castrillón, E. P. (2011, 12 5). "Revista Virtual Universidad Católica del Norte". No. 34. Retrieved 4 6, 2014, from "Revista Virtual Universidad Católica del Norte". No. 34:

http://revistavirtual.ucn.edu.co/index.php/RevistaUCN/article /viewFile/332/636

Colombia, M. d. $(2005,116)$. Colombia aprende la red del conocimiento. Retrieved 4 3, 2014, from Colombia aprende la red del conocimiento:

http://www.colombiaaprende.edu.co/html/directivos/1598/ article-99368.html

Colombia, M. d. (2014, 9 8). Banco Nacional de Recursos Educativos Retrieved 10 15, 2014, from Banco Nacional de Recursos Educativos:

http://www.colombiaaprende.edu.co/html/directivos/1598/ article-99364.html

Colombia., M. d. (2011,65). Colombia aprende la red del conocimiento. Retrieved 4 6, 2014, from Colombia aprende la red del conocimiento.:

http://www.colombiaaprende.edu.co/html/directivos/1598/ article-172369.html

comunicación., M. d. (2012). La formación de docentes en TIC. Bogotá.: EXPRECARDS C.I. - S.A.S.ISBN: 978-958-57617-0-4.

Cuervo, M. C. (2011). Objetos de aprendizaje, un estado del arte. Revista Entramado vol.7 no.1, 14 .

Cuervo., M. C. (2011. ISSN 1900-3803.). Objetos de aprendizaje, un estado del arte. Revista entramado. Vol. 7 Num.1 14.

Cuervo., M. C. (2011.). Objetos de aprendizaje un estado del arte. Revista entramado. Vol.7 No.1., 14

Diego, G. T. (2012, 1 2). Academia.Edu. Retrieved 7 1, 2014, from Academia.Edu:

http://www.academia.edu/384290/Consolidaci\%C3\%B3n_De_La _Red_Colombiana_De_Bancos_De_Objetos_De_Aprendizaje_Ret os_Y_Experiencias_En_Instituciones_De_Educaci\%C3\%B3n_Super ior?login=irlesa.sanchez@gmail.com\&email_was_taken=true

Downes, s. (2003b, January 6). learnscope.flexiblelearning.net.au/. Retrieved 810,2014 , from

http://learnscope.flexiblelearning.net.au/LearnScope/golearn .asp?Category=11\&DocumentId = 4845: http://learnscope.flexiblelearning.net.au/

Erla Morales, F. J. (2004, 8 2). Propuesta de Evaluación de Aprendizaje. Retrieved 10 15, 2014, from Propuesta de Evaluación de Aprendizaje: http://www.uoc.edu/symposia/spdece05/pdf/ID06.pdf

Friesen, N. (2004). Three Objections to Learning Objects and ELearning Standars. London UK: R. McGreal.

Galvis, A. H. (1992, 5 2). Ingeniería de Software Educativo. Retrieved 9 16, 2014, from Ingeniería de Software Educativo: https://sites.google.com/site/galvaro50/publications

García., A. (2005, 04 01). Objetos de aprendizajes. Características y repositorios. . Boletín electrónico de noticias de educación a Distancia (BENED)., p. 5.

Gibbons, A. S. (2002). The Nature and Origin of Instruction. USA: Association for Educational Communications and Technology.

González, M. C. (2005, 6 1). Colombia aprende - Diseño y desarrollo de 
materiales educativos computarizados. Retrieved 5 4, 2014, from Colombiaaprende - Diseño y desarrollo de materiales educativos computarizados: http://www.colombiaaprende.edu.co/html/mediateca/1607/ articles-106492_archivo.pdf

González, M. C. (2006, 7 2). Diseño y Desarrollo de Materiales Educativos Computarizados. Retrieved 915,2014 , from Diseño y Desarrollo de Materiales Educativos Computarizados.: http://revistavirtual.ucn.edu.co/index.php/RevistaUCN/article/ viewFile/190/365

J., O. C. (1998). ENFOQUES HIPERMEDIALES PARA EL DISEÑO Y DESARROLLO DE SOFTWARE EDUCATIVO EN EDUCACIÓN SUPERIOR. Informática Educativa - UNIANDES - LIDIE, 49-67.

Javeriana., C. U.-P. (2009). Objetos de aprendizaje. Cali.: Multimedios PUJ- ISBN: 958-8162-65-3..

Lacasa, P. (2012.). Objetos de aprendizaje y significado. Madrid.: Universidad de Alcalá.

López Guzmán, C., \& y García Peñalvo, F. J. (2004). La reutilización de recursos educativos en la Educación Superior apoyada por elearning. Revista UNAMV5 N'10 ISSN: 1067-6079, 8.

María Clara Borrero, E. C. (2010, 8 2). Una metodología para el diseño de objetos de aprendizaje. Retrieved 1015,2014 , from Una metodología para el diseño de objetos de aprendizaje.: http://objetos.univalle.edu.co/files/articulo_AMED.pdf

Marqués, P. (1995, 3 12). Metodologia para la elaboracion de software educativo en software educativo. Barcelona, La plata, Argentina: Estel.

Meta, M. M. (2010, 5 2). Importancia de los objetos de aprendizaje en la educación virtual. Retrieved 8 6, 2014, from Importancia de los objetos de aprendizaje en la educación virtual:

http://www.slideshare.net/mnieto2009/importancia-de-losobjetos-de-aprendizaje-en-la-educacion-virtual

mexico, U. n. (2012, 2 23). Repositorio Digital UNAM - Ingeniería Web (TWeb). Retrieved 4 6, 2014, from Repositorio Digital UNAM Ingeniería Web (IWeb): http://www.ptolomeo.unam.mx:8080/xmlui/bitstream/handle/ 132.248.52.100/188/A4.pdf? sequence $=4$

Mineducación. (2009, 5 30). Plan nacional de desarrollo educativo. Retrieved 4 5, 2014, from Plan nacional de desarrollo educativo: http://www.mineducacion.gov.co/1621/articles-195608_archivo _pdf.pdf

Mineducación. (2012.). Recursos educativos digitales abiertos Colombia. Bogotá.: Ministerio de educación nacional. ISBN: 978958-691-476-5.

Moral, M. E. (2005). Diseñando Objetos de Aprendizaje como facilitadores. Barcelona.: UOC.

Moral, M. E. (2005). Diseñando Objetos de Aprendizaje como facilitadores de la construcción del conocimiento. UOC, 10

nacional, M. d. (2005, 2 21). Colombia aprende. Retrieved 4 2, 2014, from Colombia aprende:

http://www.colombiaaprende.edu.co/html/directivos/1598 /articles-75230_archivo.pdf

Nacional, M. d. (2012). Colección Sistema Nacional de Innovación Educativa con uso de TIC. Bogotá: Recursos Educativos Digitales Abiertos.

Nacional., M. d. (2012). Colección: Sistema Nacional de Innovación Educativa con Uso de TIC. Bogotá.: Graficando Servicios Integrados.

Nacional., M. d. (2012). Recursos educativos digitales abiertos Colombia. Bogotá.: Creative commons. ISBN: 978-958-691-476-5.

PORTILIA, I. (2014, 2 1). EvSAL Revistas. Retrieved 10 29, 2014, from EvSAL Revistas Vol. 15, núm. 2(2014):

http://revistas.usal.es/index.php/revistatesi/article/view $/ 11887 / 12297$

Quiceno, M. S. (2009). Diseño y desarrollo de un Objeto Virtual de aprendizaje para el curso de física mecánica. Villavicencio: Ucc.

Redmutis. (2014, 8 5). Red Universitaria Mutis . Retrieved 9 27, 2014, from Red Universitaria Mutis : http://www.redmutis.org.co/

Ruffini, M. (2000, 5 23). The Interlerning Company. Retrieved 910 , 2014, from The Interlerning Company: http://www.sapioinstitute.org/research/mruffini.pdf/

Saavedra, M. F. (2012, 8 2). Plandecenal. Retrieved 4 6, 2014, from Plandecenal:

http://www.plandecenal.edu.co/html/1726/articles-310129_ recurso_1.pdf

Saavedra., M. F. (2012, 8 2). Plandecenal. Retrieved 4 1, 2014, from Plandecenal: http://www.plandecenal.edu.co/html/1726/articles-310129_ recurso_1.pdf

salud.,O.p.(2012, 5 1). Campus virtual de la salud publica. Retrieved 9 6, 2014, from Campus virtual de la salud publica: http://colombia.campusvirtualsp.org/?q=node/ 11

Suarez, O. J. (2010, 53 3). Diseño y desarrollo de objetos de arpendizaje. Retrieved 5 1, 2014, from Diseño y desarrollo de objetos de arpendizaje.:

http://www.ribiecol.org/embebidas/congreso/2008/ponencias /36.pdf

Uptc. (2014, 2 1). Tecnologías de la Información y la comunicación y Ambientes de Aprendizaje. Retrieved 8 5, 2014, from Tecnologías de la Información y la comunicación y Ambientes de Aprendizaje: http://virtual.uptc.edu.co/drupal/files/unidad5_tic/contenido/ unidad5_tics.pdf

Wiley, D. (2002). Connecting learning objects to instructional design theory: A. USA: Association for Educational Communications and Technology.

Wiley, D. A. (2003, 8 2). The Coming Collision between Automated Instruction and Social. USA: Learning Object Context \& Connections. 
